\title{
Role of Immunomarkers in the Clinicopathological Analysis of Unicystic Ameloblastoma
}

\author{
Parul Sah, ${ }^{1}$ Aparna Menon, ${ }^{1}$ Asha Kamath, ${ }^{2}$ Chetana Chandrashekar, ${ }^{1}$ \\ Sunitha Carnelio, ${ }^{1}$ and Raghu Radhakrishnan ${ }^{1}$ \\ ${ }^{1}$ Department of Oral and Maxillofacial Pathology, Manipal College of Dental Sciences, Manipal University, Manipal, \\ Karnataka 576104, India \\ ${ }^{2}$ Department of Community Medicine, Kasturba Medical College, Manipal University, Manipal, Karnataka 576104, India \\ Correspondence should be addressed to Raghu Radhakrishnan; raghu.radhakrishnan@gmail.com
}

Received 7 June 2013; Accepted 29 August 2013

Academic Editor: Gunter Haroske

Copyright (C) 2013 Parul Sah et al. This is an open access article distributed under the Creative Commons Attribution License, which permits unrestricted use, distribution, and reproduction in any medium, provided the original work is properly cited.

\begin{abstract}
Purpose. The clinical behavior of unicystic ameloblastoma varies according to its subtype. The assessment of its proliferative capacity, neovascularization, and invasiveness using relevant immunomarkers may aid in appropriate surgical therapeutic protocol. Methods. 18 cases of clinically and histologically confirmed unicystic ameloblastoma, categorized as luminal, intraluminal, or mural subtypes, were analyzed retrospectively. Immunomarkers such as Ki-67, CD34, MMP-2, and MMP-9 were studied to evaluate their behavior. Results. Labeling index of Ki-67 was $4.25 \%$ in the intraluminal subtype, compared with $2.14 \%$ in the luminal and $4.04 \%$ in the mural variant $(P=0.3)$. CD34 immunostaining was significantly higher in the mural variant (43 per high power field) than the other two subtypes $(P=0.04)$. MMP-2 and MMP-9 were strongly expressed in mural, moderately in intraluminal, and weakly to absent in luminal variant. Conclusions. High proliferative index, angiogenesis, and protease activity in the mural ameloblastoma, ascertained by the expression of these markers, confirm its aggressive phenotype. The intraluminal and luminal subtype exhibiting decreased expression are compatible with their indolent clinical behavior.
\end{abstract}

\section{Introduction}

Ameloblastoma is a slow growing, locally invasive, epithelial odontogenic tumor of the jaws with a high rate of recurrence if not removed adequately, but with virtually no tendency to metastasize [1]. Among the intraosseous ameloblastomas, 5$22 \%$ are the clinicopathological subtype known as unicystic ameloblastoma [2]. The term unicystic ameloblastoma refers to those lesions that display the clinical, radiographic, or gross features of a jaw cyst, but on histologic examination they reveal an epithelial lining composed focally or entirely of ameloblastomatous epithelium [3-5]. In contrast to the aggressive growth and prolific local invasion of its solid counterpart, the unicystic ameloblastoma is considered less destructive and more amenable to conservative treatment [6].

Unicystic ameloblastoma has been categorized into the luminal, intraluminal, and mural subtypes $[3,7]$ based on the growth pattern of the ameloblastomatous epithelium. While ameloblastomatous epithelium is confined to the luminal surface in the luminal type, it projects into the lumen as a polypoid exophytic mass in the intraluminal type and grows inward into the fibrous wall of the cyst in the mural type [2]. Grossing the whole specimen is crucial for subtyping a unicystic ameloblastoma as the variation in the lining epithelium portends its biologic behavior, thereby necessitating a case-specific treatment approach [8]. This variation in clinical behavior is due to the differential growth pattern of the lining epithelium with regard to the invasive and proliferative capacity of the three subtypes.

The nuclear antigen, Ki-67, is a widely used cell proliferation marker used in both the normal and neoplastic tissue. Evaluation of proliferative potential of ameloblastomatous epithelium in the cystic lining reflects the tumor behavior and tendency towards recurrence [9]. Microvessel density (MVD) is considered a bona fide predictor of tumor growth and metastatic potential [10]. Assessment of MVD using a pan endothelial marker, CD34, is a significant indicator of tumor invasion and postoperative recurrence. Matrix 
metalloproteinases (MMPs), a family of zinc- and calciumdependent proteolytic enzymes [11], mediate extracellular matrix destruction, particularly during tumor invasion and metastasis. MMP-2 and MMP-9 are of added relevance owing to their ability to degrade collagen IV, the main basement membrane component [11-13].

The use of these immunohistochemical markers in solid ameloblastoma has confirmed its local invasiveness, high proliferative capacity, and increased neovascularisation [11, 1315]. Consequently, the utilization of these immunomarkers to address the biologic behavior of subtypes of unicystic ameloblastoma is pertinent so as to embark on the most suitable treatment protocol. The aim of this study was to investigate and compare the expression of MMP-2, MMP-9, $\mathrm{Ki}-67$, and CD34 in the three variants of unicystic ameloblastoma.

\section{Materials and Methods}

2.1. Patients and Tissue Samples. The study group comprised of eighteen cases of unicystic ameloblastoma retrieved from the departmental archives. Multiple sections taken at several levels for each case were reviewed following routine staining. Based on the characteristics of the lining epithelium, cases were grouped into luminal $(n=6)$, intraluminal $(n=6)$, and mural $(n=6)$ subtype. In cases showing features of more than one subtype, the more aggressive subtype was considered. Sections that showed pronounced inflammation in the connective tissue were excluded. Due to the retrospective nature of this study, it was granted an exemption in writing by the Institutional Ethics Committee, Manipal University.

2.2. Immunohistochemistry. Immunohistochemical staining of the tissue sections from each of the cases selected was stained using streptavidin-biotin method. In brief, $4 \mu \mathrm{m}$ sections was mounted on 3-aminopropyltriethoxysilane (APES) coated slides, deparaffinized through a series of xylene baths, and then rehydrated in graded alcohols. Antigen retrieval was performed in a pressure cooker for 15 minutes in citrate buffer ( $\mathrm{pH}$ 6.0) for Ki-67 and Tris-EDTA buffer ( $\mathrm{pH} 9.0$ ) for MMP-2, MMP-9, and CD34. Sections were immersed in peroxidase block and protein block (Novolink Polymer Detection System, Novocastra) for 30 minutes each. Slides were then incubated with primary antibodies against MMP9 (clone 15W2, Novocastra) diluted 1:80, MMP-2 (clone 17B11, Novocastra) diluted 1:20, Ki-67 (clone BGX-297, Biogenex) diluted 1:40, and CD34 (clone QBend/10, Biogenex) diluted 1:60 for one hour. The sections were subsequently washed in Tris-buffered saline and incubated with secondary biotinylated antibody and streptavidin-biotin peroxidase complex (Novolink Polymer Detection System, Novocastra) for 30 minutes each. Diaminobenzidine (DAB) was used as the chromogen and the sections were counterstained with Mayer's haematoxylin.

2.3. Immunostaining Evaluation. Ki 67 expression was estimated in five fields from each case, selected randomly at a magnification of $\times 200$. Ki-67 positive cells showed a distinct brown nuclear stain and accordingly the Ki-67 labeling index (Ki-67 LI) was estimated as percentage of cells with positive nuclei [9]. FIJI, an open source image-processing package based on ImageJ, was used to assess the LI. The microvessel density (MVD) was quantified using CD34 to assess the neovascularisation. A microvessel was defined as an endothelial marker-stained cell or cluster that was separate from adjacent cells or clusters and present within the vicinity of the tumor but not in necrotic or sclerotic zones [10]. MVD was quantified in three areas with the highest neovascularization (hotspots), found by scanning the tumor sections at low power using an Olympus BX41 brightfield microscope (Olympus, Tokyo, Japan). Microvessels were counted in each of the three fields at $\times 200$ magnification and the mean density was reported. The field size for $\times 200$ magnification was approximately $1.1 \mathrm{~mm}^{2}$. MVD was expressed as the mean number of microvessels per high power field (HPF) [16]. The immunohistochemical expression of gelatinases, MMP2, and MMP-9 was assessed based on the criteria put forth by Yoon et al. [17]. The percentages of immunopositive cells were scored as $0=0 \%, 1=10 \%, 2=$ more than $10 \%$ and less than $50 \%$, and $3=$ more than $50 \%$. The staining intensity was recorded as $0=$ negative, $1=$ weak, $2=$ moderate, and $3=$ strong positive. The final immunoscore for MMP-2 and 9 was calculated by multiplying the percentage score and intensity score and graded as negative $(-)$ for 0 , weak positive $(+)$ for 1 and 2 , moderate positive $(++)$ for 3 and 4 , and strong positive $(+++)$ for 6 and 9.

2.4. Statistical Analysis. The data obtained was statistically analyzed with the statistical software program SPSS (version 15.0). The statistical significance of variation in Ki-67 LI and MVD between the three subtypes was analyzed using the Kruskal-Wallis test. $P$ values less than 0.05 were considered to indicate statistical significance. The comparison of MMP2 and MMP-9 expression between the three subtypes was performed using the chi-square test.

\section{Results}

The age of the patients in our series of cases of unicystic ameloblastoma ranged from 13 years to 50 years. Most of the patients in our series were men, with a male to female ratio of $13: 5$. The predominant site of occurrence was the mandible, with 17 out of 18 cases present either in the molar-ramus region or in the parasymphysis. All the lesions presented as well-defined radiolucencies, ranging in size from 2.5 to $6.6 \mathrm{~cm}$. Other radiographic features included cortical thinning and perforation (8/18 cases), tooth displacement (8/18 cases), and root resorption (14/18 cases) (Figure 1$)$. Since radical treatment (segmental resection or hemimandibulectomy) was performed for the majority of the cases, recurrence could not be assessed. The clinicopathological information for all selected cases is summarized in Table 1.

The Ki-67 LI was $4.25 \%$ and $4.04 \%$ in the intraluminal and mural variants, respectively, compared to $2.14 \%$ in the luminal variant $(P=0.3)$ (Figures $2(\mathrm{a})-2(\mathrm{c}))$. The median MVD was 25.45 per HPF in the luminal variant, 36.8 per HPF 
TABLE 1: Clinicopathological details of selected cases.

\begin{tabular}{|c|c|c|c|c|c|c|c|c|c|}
\hline \multirow[b]{2}{*}{$\begin{array}{l}\text { Case } \\
\text { no. }\end{array}$} & \multirow[b]{2}{*}{$\begin{array}{l}\text { Age (in } \\
\text { years) }\end{array}$} & \multirow[b]{2}{*}{ Sex } & \multirow[b]{2}{*}{ Site } & \multirow[b]{2}{*}{ Treatment done } & \multicolumn{5}{|c|}{ Radiographic features } \\
\hline & & & & & $\begin{array}{l}\text { Size in } \\
\mathrm{cm}\end{array}$ & $\begin{array}{l}\text { Marginal } \\
\text { definition }\end{array}$ & $\begin{array}{l}\text { Cortical } \\
\text { thinning and } \\
\text { perforation }\end{array}$ & $\begin{array}{c}\text { Tooth } \\
\text { displacement }\end{array}$ & $\begin{array}{c}\text { Root } \\
\text { resorption }\end{array}$ \\
\hline 1 & 24 & $\mathrm{~F}$ & Right mandible & Hemimandibulectomy & $5-6$ & Well defined & Present & Present & Present \\
\hline 2 & 50 & $\mathrm{~F}$ & $\begin{array}{l}\text { Right anterior } \\
\text { maxilla }\end{array}$ & Hemimandibulectomy & 3.0 & Well defined & Absent & Absent & Present \\
\hline 3 & 32 & $\mathrm{~F}$ & $\begin{array}{l}\text { Left mandible } \\
\text { (angle and } \\
\text { ramus) }\end{array}$ & Segmental resection & 6.0 & Well defined & Absent & Absent & Present \\
\hline 4 & 35 & $\mathrm{M}$ & $\begin{array}{l}\text { Left mandible } \\
\text { (body) }\end{array}$ & Segmental resection & 3.4 & Well defined & Present & Absent & Absent \\
\hline 5 & 40 & $\mathrm{M}$ & $\begin{array}{l}\text { Right mandible } \\
\text { (body and } \\
\text { ramus) }\end{array}$ & Hemimandibulectomy & $4-5$ & Well defined & Absent & Absent & Present \\
\hline 6 & 51 & $\mathrm{M}$ & $\begin{array}{l}\text { Left anterior } \\
\text { mandible }\end{array}$ & Hemimandibulectomy & 3.7 & Well defined & Present & Absent & Present \\
\hline 7 & 47 & $\mathrm{M}$ & $\begin{array}{l}\text { Anterior } \\
\text { mandible }\end{array}$ & Enucleation and curettage & 3.56 & Well defined & Present & Absent & Present \\
\hline 8 & 25 & $\mathrm{~F}$ & $\begin{array}{l}\text { Right mandible } \\
\text { (parasymphysis) }\end{array}$ & Segmental resection & 6.2 & Well defined & Present & Present & Present \\
\hline 9 & 32 & M & $\begin{array}{l}\text { Right mandible } \\
\text { (parasymphysis) }\end{array}$ & Hemimandibulectomy & $3-4$ & Well defined & Absent & Present & Present \\
\hline 10 & 13 & $\mathrm{M}$ & $\begin{array}{l}\text { Right posterior } \\
\text { mandible }\end{array}$ & Hemimandibulectomy & 4.3 & Well defined & Absent & Present & Present \\
\hline 11 & 39 & $\mathrm{M}$ & $\begin{array}{l}\text { Right mandible } \\
\text { (parasymphysis) }\end{array}$ & Hemimandibulectomy & 3.0 & Well defined & Absent & Present & Present \\
\hline 12 & 35 & $\mathrm{M}$ & $\begin{array}{l}\text { Anterior } \\
\text { mandible }\end{array}$ & Hemimandibulectomy & 2.5 & Well defined & Absent & Absent & Absent \\
\hline 13 & 20 & $\mathrm{M}$ & $\begin{array}{l}\text { Right mandible } \\
\text { (parasymphysis) }\end{array}$ & Segmental resection & 5.0 & Well defined & Present & Present & Present \\
\hline 14 & 24 & $\mathrm{~F}$ & $\begin{array}{l}\text { Right mandible } \\
\text { (angle and } \\
\text { ramus) }\end{array}$ & Hemimandibulectomy & 6.6 & Well defined & Present & Present & Present \\
\hline 15 & 32 & $\mathrm{M}$ & $\begin{array}{l}\text { Right mandible } \\
\text { (parasymphysis) }\end{array}$ & Hemimandibulectomy & 4.0 & Well defined & Absent & Present & Present \\
\hline 16 & 20 & $\mathrm{M}$ & $\begin{array}{l}\text { Right mandible } \\
\text { (parasymphysis) }\end{array}$ & Hemimandibulectomy & 2.3 & Well defined & Absent & Absent & Absent \\
\hline 17 & 40 & $\mathrm{M}$ & $\begin{array}{l}\text { Right mandible } \\
\text { (body and } \\
\text { ramus) }\end{array}$ & Segmental resection & 1.5 & Well defined & Absent & Absent & Absent \\
\hline 18 & 40 & $\mathrm{M}$ & $\begin{array}{l}\text { Right mandible } \\
\text { (parasymphysis) }\end{array}$ & Segmental resection & 1.78 & Well defined & Present & Absent & Present \\
\hline
\end{tabular}

in the intraluminal subtype, and 43 per HPF in the mural variant (Figures $2(\mathrm{~d})-2(\mathrm{f}))$. A statistically significant $(P=$ 0.04 ) difference in MVD was seen when the three subtypes were compared.

MMP-2 expression was seen in the cytoplasm of ameloblastomatous epithelium and stellate reticulum-like cells as well as in cells of the surrounding stroma (Figures 3(a)-3(c)). Reactivity in the luminal variant was predominantly weak in the lining epithelium. The intraluminal variant showed uniformly moderate reactivity in all the 6 cases. The expression in the mural variant was strong positive, in 5 out of 6 cases. MMP-9 expression was similarly identified in both tumor cells and stromal cells (Figures 3(d)-3(f)). The luminal variant showed equal distribution of negative 


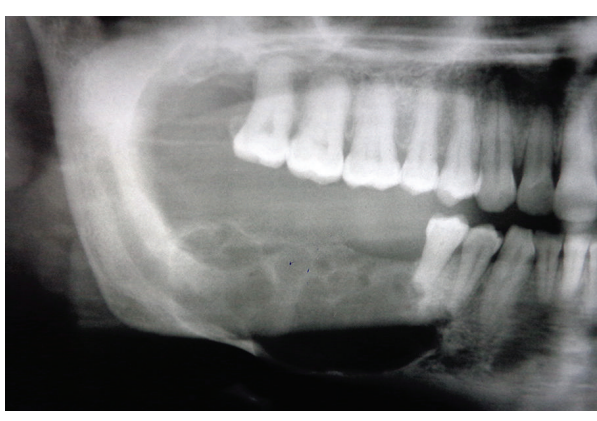

(a)

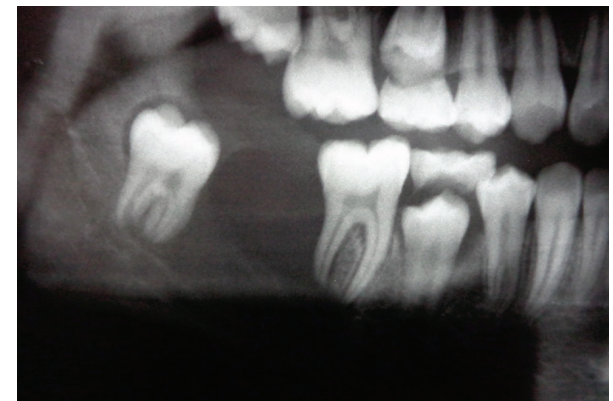

(b)

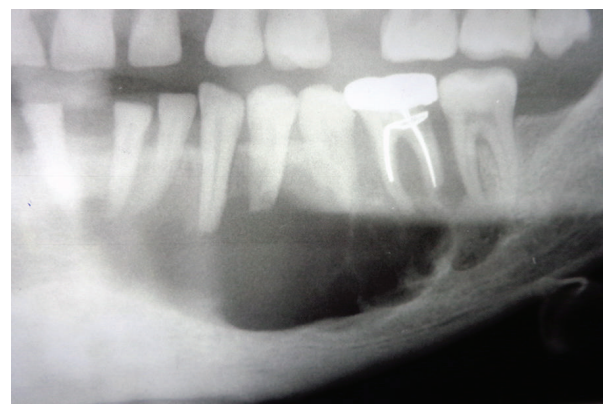

(c)

FIGURE 1: Radiographic presentations of unicystic ameloblastoma: (a) multilocular radiolucency with scalloped border, cortical thinning, perforation, and resorption; (b) well-defined unilocular radiolucency; (c) multilocular radiolucency with root resorption.

TABLE 2: Immunoprofiles of Ki-67, CD34, MMP-9, and MMP-2 in cystic ameloblastoma.

\begin{tabular}{|c|c|c|c|c|c|}
\hline Pathological type & Case no. & $\mathrm{Ki}-67 \mathrm{LI}^{* *}(\%)$ & $\mathrm{MVD}^{* * *}$ (per HPF) & MMP-2 expression* & MMP-9 expression* \\
\hline \multirow{6}{*}{ Luminal } & 2 & 5.59 & 18.3 & - & - \\
\hline & 7 & 6.25 & 29.6 & + & + \\
\hline & 8 & 0.6 & 21.3 & + & - \\
\hline & 10 & 2.33 & 32.3 & - & - \\
\hline & 12 & 1.95 & 31.6 & + & + \\
\hline & 16 & 1.45 & 21 & + & + \\
\hline \multirow{6}{*}{ Intraluminal } & 3 & 3.95 & 36.3 & ++ & ++ \\
\hline & 4 & 6.2 & 37.3 & ++ & ++ \\
\hline & 6 & 1.85 & 42.3 & ++ & ++ \\
\hline & 9 & 4.56 & 20.3 & ++ & ++ \\
\hline & 17 & 2.85 & 47.3 & ++ & ++ \\
\hline & 18 & 5.97 & 21 & ++ & ++ \\
\hline \multirow{6}{*}{ Mural } & 1 & 9.13 & 52 & ++ & ++ \\
\hline & 5 & 4.78 & 47 & +++ & +++ \\
\hline & 11 & 2.72 & 22 & +++ & ++ \\
\hline & 13 & 4.43 & 54 & +++ & +++ \\
\hline & 14 & 3.08 & 37 & +++ & +++ \\
\hline & 15 & 3.65 & 39 & +++ & +++ \\
\hline
\end{tabular}

${ }^{*}$ Immunohistochemical reactivity for MMP-2 and MMP-9: (-) negative, $(+)$ weak positive, $(++)$ moderate positive, and (+++) strong positive. ${ }^{* *}$ Ki-67 LI: Ki-67 labeling index.

${ }^{* * *}$ MVD: microvessel density. 


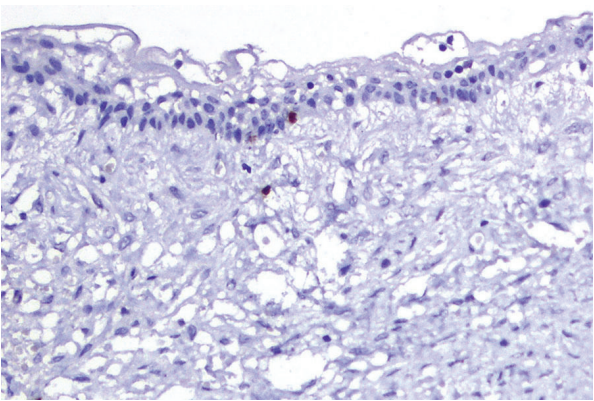

(a)

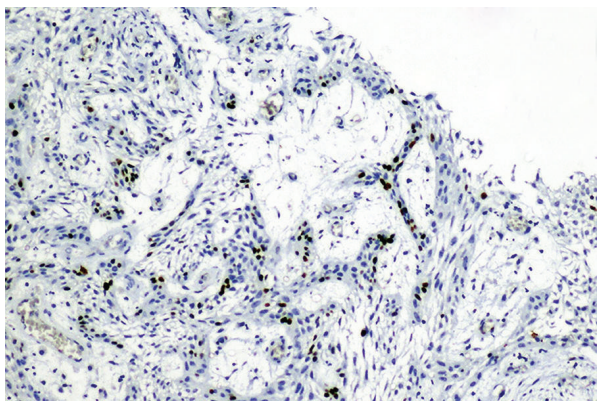

(c)

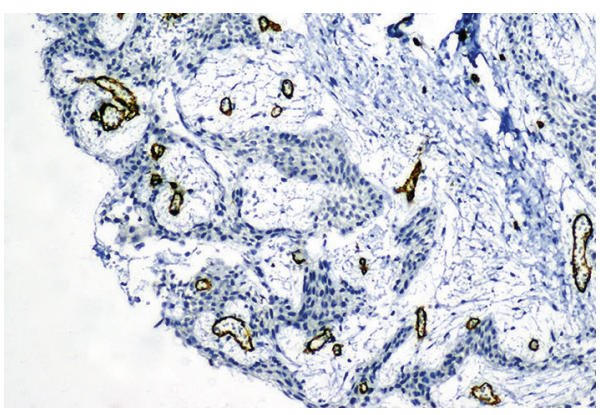

(e)

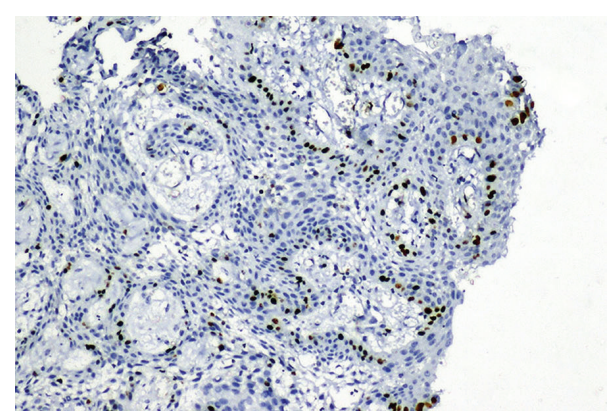

(b)

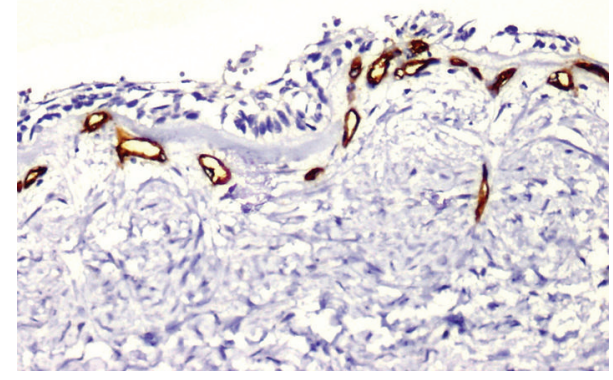

(d)

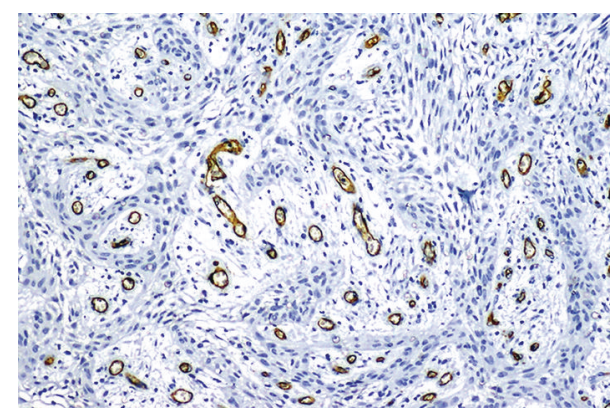

(f)

FIGURE 2: Nuclear expression of Ki-67 in unicystic ameloblastoma subtypes (immunoperoxidase): (a) luminal $(\times 200)$; (b) intraluminal $(\times 100)$; (c) mural $(\times 100)$. Unicystic ameloblastoma subtypes showing CD34 expression (immunoperoxidase); (d) luminal ( $\times 200)$; (e) intraluminal $(\times 200)$; (f) mural $(\times 100)$.

and weak MMP-9 reactivity. Cases of intraluminal ameloblastoma showed moderate reactivity. In mural ameloblastoma, reactivity was mainly strong positive, with 2 cases scored as moderate. A strong correlation could be established between MMP-2 and MMP-9 staining with regard to immunoscore. Table 2 summarizes the immunohistochemical findings for Ki-67, CD34, MMP-2, and MMP-9 in the subtypes of unicystic ameloblastoma.

\section{Discussion}

Unicystic ameloblastoma as a subtype of intraosseous ameloblastoma has long been delineated as a prognostically distinct entity [3]. It occurs in younger patients, predominantly in the second to third decade, with the mean age at presentation reported as 25.5 years [4]. The site of preference is the mandible, with the mandible to maxilla ratio being 13.1:3 according to one study. The majority of tumors are located in the third molar region, and $50-80 \%$ of cases are associated with an impacted tooth [2]. The histopathological features vary, and each of the subtypes may exist alongside a flat, odontogenic epithelial lining closely resembling a jaw cyst [18].

Unicystic ameloblastomas exhibit a recurrence rate of $6.7-35.7 \%$ in contrast to solid ameloblastomas, where it can be as high as $70-80 \%$ with initial conservative therapy [19]. The mural variant, however, has recurrence rates similar to those of the conventional type if treatment is limited to enucleation and curettage. When the tumor infiltrates the cystic wall, it is in principle believed to have a solid component which overshadows the cystic component with respect to treatment planning [19]. For this reason, Ackermann et al. suggested a radical surgical approach when treating mural ameloblastoma [7].

The demonstration of a higher median value of Ki-67 in the intraluminal and mural variants compared to the luminal 


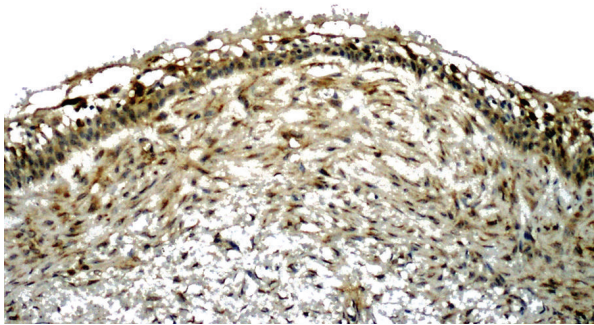

(a)

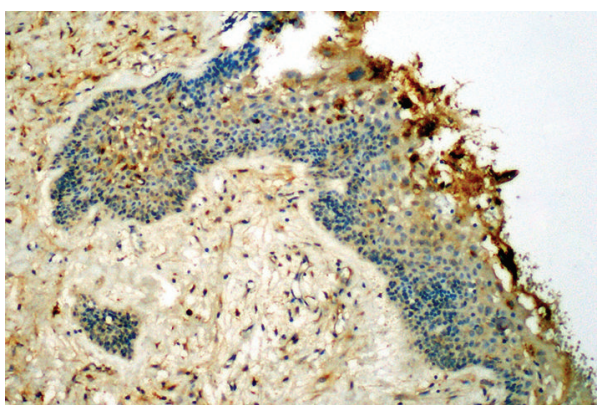

(c)

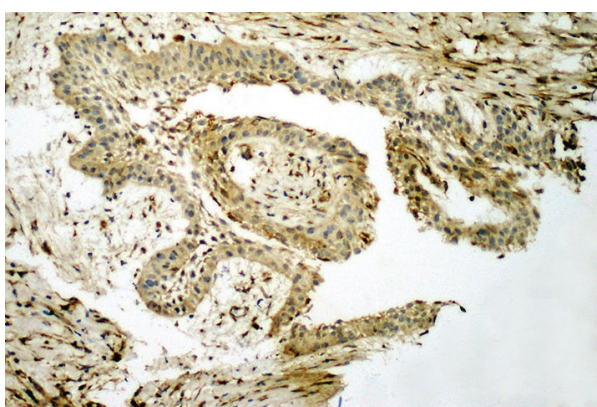

(e)

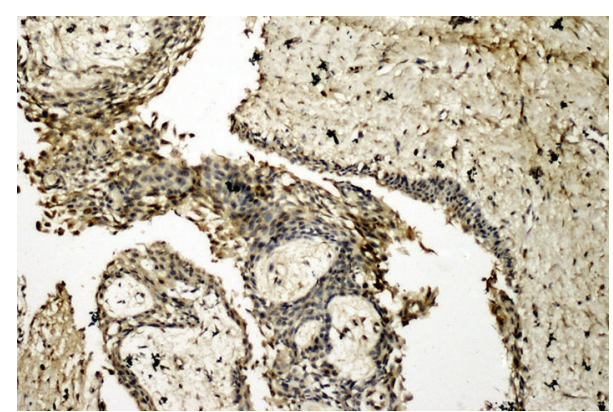

(b)

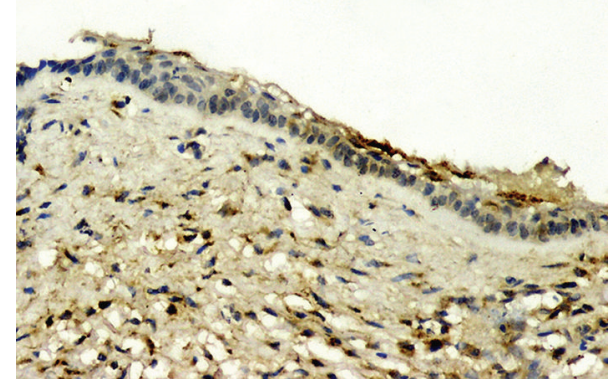

(d)

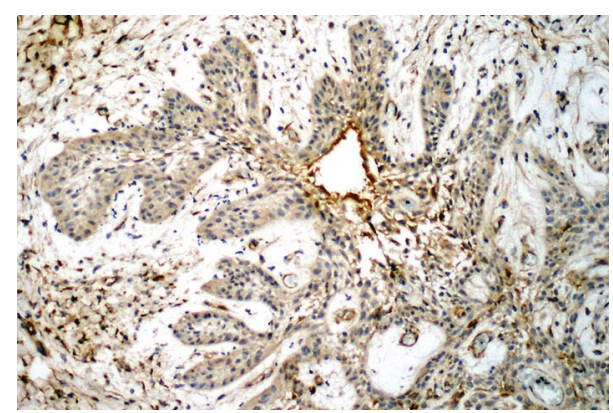

(f)

FIGURE 3: MMP 2 immunostaining in variants of unicystic ameloblastoma (immunoperoxidase): (a) luminal $(\times 100)$; (b) intraluminal $(\times 100)$; (c) mural ( $\times 100)$. MMP-9 expression in unicystic ameloblastoma (immunoperoxidase): (d) luminal $(\times 200)$; (e) intraluminal $(\times 100)$; (f) mural $(\times 100)$.

variant in our study is in accordance with investigations by Meer et al. [20] and Rizzardi et al. [21]. Similarly, Li et al. reported the Ki-67 LI to be highest in the mural variant and significantly lower in the other two subtypes [22]. In contrast to these findings, Bologna-Molina et al. in their study found Ki-67 LI to be highest in the luminal variant followed by the intraluminal and mural variants [23]. These results indicate a differential proliferative capacity of the ameloblastomatous lining in each of the subtypes. Assessment of Ki-67 LI in all the cases of unicystic ameloblastoma may be crucial in elucidating its disparate biological behavior. The high Ki-67 $\mathrm{LI}$ in the mural and the intraluminal variant compared to the luminal variant confirms its higher proliferative potential. LI in the intraluminal variant comparable to the mural variants may be due to the presence of exophytic nodules in the cystic lining, which often show features of typical plexiform presentation.
The growth, survival, and expansion of a tumor are largely dependent on the vascular system recruited by the neoplasm [24]. Although several studies have investigated the MVD in solid ameloblastomas [14, 24, 25], this is the first study where it has been studied in the subtypes of unicystic ameloblastoma. Neovascularization may be related to the variation in biological behaviors of these lesions and reflects their clinical characteristics to some degree. We observed an elevated median MVD in the mural variant, followed by intraluminal and luminal. This finding is in accordance with Hande et al. [26], who found MVD to be higher in unicystic ameloblastoma that had a mural component. The increase in MVD in the mural variant suggests that the increased growth potential of the ameloblastomatous lining is the result of elevated neovascularization or angiogenesis in this variant [24]. The increase in MVD values from luminal to mural variant confirms that the mural variant is more belligerent 
and prone to recurrence, while the intraluminal and luminal variants are thus amenable to treatment by enucleation and curettage. The appraisal of the tumor microvasculature may thus provide important prognostic information about the unicystic ameloblastoma subtypes.

Matrix metalloproteinases are highly homologous $\mathrm{Zn++}$ endopeptidases secreted by both tumor and stromal cells [27], which play an essential role in numerous physiological processes, including embryonic development, reproduction, inflammation, and wound healing [28]. MMP-2 and MMP9 are known to degrade several ECM proteins including type IV collagen, gelatin, and fibronectin, which are essential components of the basement membrane. MMP-9 has also been established as a regulator of neovascularisation by activating fibroblast growth factor (FGF), vascular endothelial growth factor (VEGF), and transforming growth factor beta (TGF$\beta$ ) [27]. MMP-2 and MMP-9 mediate tumor growth and invasion by regulating events like neoangiogenesis, apoptosis, invasion, and metastasis via cleavage of the growth factor receptor precursors, cell adhesion molecules, and disintegration of the basement membrane. The degradative capacity of these MMPs enables neoplastic cells to migrate and colonize host tissues [29].

Numerous studies have concluded that MMP-2/MMP-9 expression is correlated with enhanced tumor growth and invasive phenotype in solid ameloblastomas [11-13, 17]. Our results show that unicystic ameloblastoma expresses MMP2 and MMP-9 in both the ameloblastomatous and stromal cells, particularly strong in the mural subtype. Epithelial cells, fibroblasts, macrophages, plasma cells, lymphocytes, and neutrophils produced MMPs in the stroma, and their expression was possibly due to the neoplastically altered lining epithelium [30]. These MMPs degrade the bone matrix and release mitogenic factors, thereby increasing tumor proliferation [12]. MMP-2 and MMP-9 expression in the intraluminal and luminal variants were moderate and negative to weak, respectively, which confirms their low invasive potential and justifies their treatment by conservative methods. The expression of MMP-2 and MMP-9 in our study appears to correlate with the clinical behavior of the three subtypes of unicystic ameloblastoma.

\section{Conclusion}

This study thus provides a molecular rationale for the difference in behavior between the three subtypes of cystic ameloblastoma. The high proliferative potential, elevated neoangiogenesis, and increased expression of gelatinases in mural ameloblastoma explicate its clinically belligerent behavior and substantiate its treatment by radical resection. In contrast, luminal and intraluminal variants have shown lower expression of these markers, thus explaining their more indolent presentation and confirming the use of enucleation and curettage as adequate treatment. Considering the fact that MVD correlated better with the clinical behavior of unicystic ameloblastoma subtypes than Ki-67 LI, MVD appears to be a more sensitive indicator of tumor behavior than Ki67 LI. In light of these observations, the use of aggressive treatment modalities in luminal and intraluminal variants seems unwarranted and should be avoided. However, these results are to be considered as preliminary observations of a case series of unicystic ameloblastoma. The assessment of biologic behavior of its varied subtypes using these markers in a bigger sample size with careful followup after conservative and radical surgery is deemed necessary for validating the behavior of unicystic ameloblastoma in a case-specific manner.

\section{References}

[1] D. G. Gardner, K. Heikinheimo, M. Shear, H. P. Philipsen, and H. Coleman, "Ameloblastomas," in World Health Organization Classification of Tumors. Pathology and Genetics. Head and Neck Tumours, L. Barnes, J. W. Eveson, P. Reichart, and D. Sidransky, Eds., pp. 296-300, IARC Press, Lyon, France, 2005.

[2] H. P. Philipsen and P. A. Reichart, "Unicystic ameloblastoma: a review of 193 cases from the literature," Oral Oncology, vol. 34, no. 5, pp. 317-325, 1998.

[3] L. Robinson and M. G. Martinez, "Unicystic ameloblastoma: a prognostically distinct entity," Cancer, vol. 40 , no. 5, pp. $2278-$ 2285, 1977.

[4] T. J. Li, Y. T. Wu, S. F. Yu, and G. Y. Yu, "Unicystic ameloblastoma: a clinicopathologic study of 33 Chinese patients," The American Journal of Surgical Pathology, vol. 24, no. 10, pp. 13851392, 2000.

[5] R. A. Vickers and R. J. Gorlin, "Ameloblastoma: delineation of early histopathologic features of neoplasia," Cancer, vol. 26, no. 3, pp. 699-710, 1970.

[6] Z. Chaudhary, V. Sangwan, U. S. Pal, and P. Sharma, "Unicystic ameloblastoma: a diagnostic dilemma," National Journal of Maxillofacial Surgery, vol. 2, no. 1, pp. 89-92, 2011.

[7] G. L. Ackermann, M. Altini, and M. Shear, "The unicystic ameloblastoma: a clinicopathological study of 57 cases," Journal of Oral Pathology, vol. 17, no. 9-10, pp. 541-546, 1988.

[8] R. S. Ramesh, S. Manjunath, T. H. Ustad, S. Pais, and K. Shivakumar, "Unicystic ameloblastoma of the mandible-an unusual case report and review of literature," Head and Neck Oncology, vol. 2, article 1, 2010.

[9] A. Abdel-Aziz and M. M. Amin, "EGFR, CD10 and proliferation marker Ki67 expression in ameloblastoma: possible role in local recurrence," Diagnostic Pathology, vol. 7, no. 1, p. 14, 2012.

[10] S. Sharma, M. C. Sharma, and C. Sarkar, "Morphology of angiogenesis in human cancer: a conceptual overview, histoprognostic perspective and significance of neoangiogenesis," Histopathology, vol. 46, no. 5, pp. 481-489, 2005.

[11] H. Kumamoto, K. Yamauchi, M. Yoshida, and K. Ooya, "Immunohistochemical detection of matrix metalloproteinases (MMPs) and tissue inhibitors of metalloproteinases (TIMPs) in ameloblastomas," Journal of Oral Pathology and Medicine, vol. 32, no. 2, pp. 114-120, 2003.

[12] J. J. Pinheiro, V. M. Freitas, A. I. Moretti, A. G. Jorge, and R. G. Jaeger, "Local invasiveness of ameloblastoma. Role played by matrix metalloproteinases and proliferative activity," Histopathology, vol. 45, no. 1, pp. 65-72, 2004.

[13] A. S. Siqueira, M. R. Carvalho, A. C. Monteiro, V. M. Freitas, R. G. Jaeger, and J. J. Pinheiro, "Matrix metalloproteinases, TIMPs and growth factors regulating ameloblastoma behaviour," Histopathology, vol. 57, no. 1, pp. 128-137, 2010. 
[14] S. Seifi, S. Shafaie, and S. Ghadiri, "Microvessel density in follicular cysts, keratocystic odontogenic tumours and ameloblastomas," Asian Pacific Journal of Cancer Prevention, vol. 12, no. 2, pp. 351-356, 2011.

[15] A. Florescu, C. Simionescu, R. Ciurea, and A. Pitru, "P53, Bcl2 and Ki67 immunoexpression in follicular solid ameloblastomas," Romanian Journal of Morphology and Embryology, vol. 53, no. 1, pp. 105-109, 2012.

[16] N. Weidner, J. P. Semple, W. R. Welch, and J. Folkman, “Tumor angiogenesis and metastasis-correlation in invasive breast carcinoma," The New England Journal of Medicine, vol. 324, no. 1, pp. 1-8, 1991.

[17] H.-J. Yoon, B. C. Jo, W. J. Shin et al., "Comparative immunohistochemical study of ameloblastoma and ameloblastic carcinoma," Oral Surgery, Oral Medicine, Oral Pathology, Oral Radiology and Endodontology, vol. 112, no. 6, pp. 767-776, 2011.

[18] V. J. Paikkatt, S. Sreedharan, and V. P. Kannan, "Unicystic ameloblastoma of the maxilla: a case report," Journal of Indian Society of Pedodontics and Preventive Dentistry, vol. 25, no. 2, pp. 106-110, 2007.

[19] C. C. Black, R. R. Addante, and C. A. Mohila, "Intraosseous ameloblastoma," Oral Surgery, Oral Medicine, Oral Pathology, Oral Radiology and Endodontology, vol. 110, no. 5, pp. 585-592, 2010.

[20] S. Meer, J. S. Galpin, M. Altini, H. Coleman, and H. Ali, "Proliferating cell nuclear antigen and Ki67 immunoreactivity in ameloblastomas," Oral Surgery, Oral Medicine, Oral Pathology, Oral Radiology, and Endodontics, vol. 95, no. 2, pp. 213-221, 2003.

[21] C. Rizzardi, P. Leocata, L. Ventura et al., "Apoptosis-related factors (TRAIL, DR4, DR5, DcRl, DcR2, apoptotic cells) and proliferative activity in ameloblastomas," Anticancer Research, vol. 29, no. 4, pp. 1137-1142, 2009.

[22] T.-J. Li, R. M. Browne, and J. B. Matthews, "Expression of proliferating cell nuclear antigen (PCNA) and Ki-67 in unicystic ameloblastoma," Histopathology, vol. 26, no. 3, pp. 219-228, 1995.

[23] R. Bologna-Molina, A. Mosqueda-Taylor, N. Molina-Frechero, A. D. Mori-Estevez, and G. Sánchez-Acuña, "Comparison of the value of PCNA and Ki-67 as markers of cell proliferation in ameloblastic tumors," Medicina Oral, Patología Oral y Cirugía Bucal, vol. 18, no. 2, pp. el74-e179, 2013.

[24] M. Alaeddini, S. Salah, F. Dehghan, N. Eshghyar, and S. Etemad-Moghadam, "Comparison of angiogenesis in keratocystic odontogenic tumours, dentigerous cysts and ameloblastomas," Oral Diseases, vol. 15, no. 6, pp. 422-427, 2009.

[25] L. J. Jiang, C. K. Shao, D. He, W. G. Li, X. Z. Wu, and D.-Z. Cai, "Correlations of extrocellular matrix metalloproteinase inducer and microvessel density to invasiveness of ameloblastoma," $\mathrm{Ai}$ Zheng, vol. 27, no. 12, pp. 1263-1266, 2008.

[26] A. H. Hande, A. R. Gadbail, A. M. Sonone, M. S. Chaudhary, V. Wadhwan, and A. Nikam, "Comparative analysis of tumour angiogenesis in solid multicystic and unicystic ameloblastoma by using CD 105 (endoglin)," Archives of Oral Biology, vol. 56, no. 12, pp. 1635-1640, 2011.

[27] M. Björklund and E. Koivunen, "Gelatinase-mediated migration and invasion of cancer cells," Biochimica et Biophysica Acta, vol. 1755, no. 1, pp. 37-69, 2005.

[28] G. Klein, E. Vellenga, M. W. Fraaije, W. A. Kamps, and E. S. de Bont, "The possible role of matrix metalloproteinase (MMP)-2 and MMP-9 in cancer, e.g. acute leukemia," Critical Reviews in Oncology/Hematology, vol. 50, no. 2, pp. 87-100, 2004.
[29] H. D. Foda and S. Zucker, "Matrix metalloproteinases in cancer invasion, metastasis and angiogenesis," Drug Discovery Today, vol. 6, no. 9, pp. 478-482, 2001.

[30] Á. C. Henriques, M. G. Vasconcelos, H. C. Galvão, L. B. De Souza, and R. D. A. Freitas, "Comparative analysis of the immunohistochemical expression of collagen IV, MMP-9, and TIMP-2 in odontogenic cysts and tumors," Oral Surgery, Oral Medicine, Oral Pathology, Oral Radiology and Endodontology, vol. 112, no. 4, pp. 468-475, 2011. 


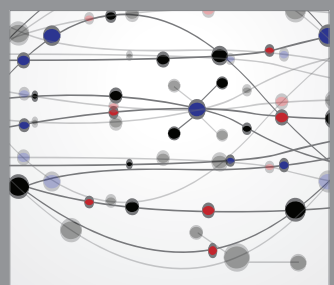

The Scientific World Journal
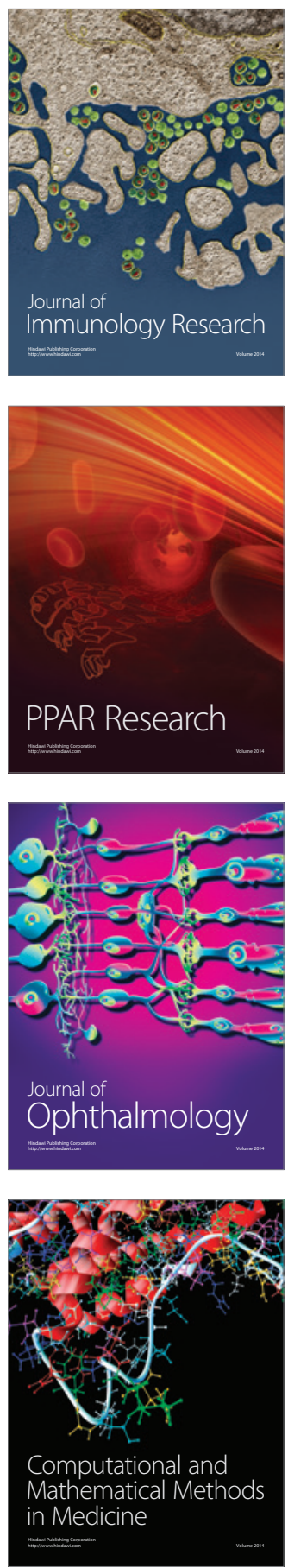

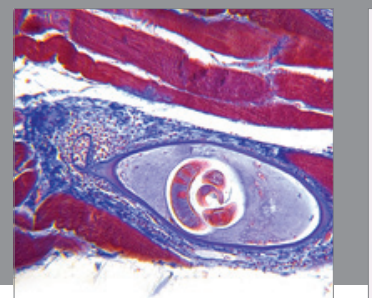

Gastroenterology

Research and Practice
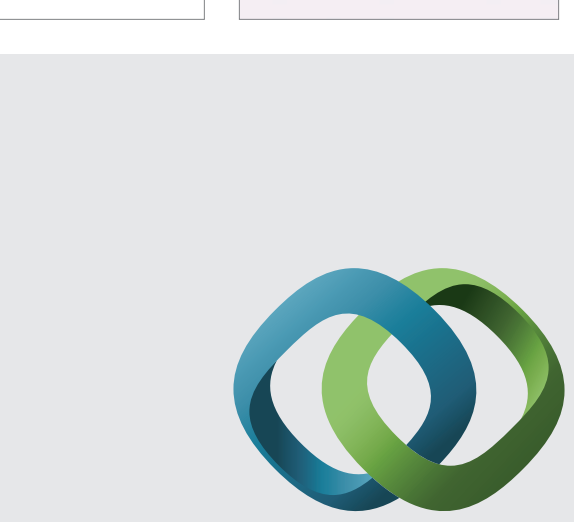

\section{Hindawi}

Submit your manuscripts at

http://www.hindawi.com
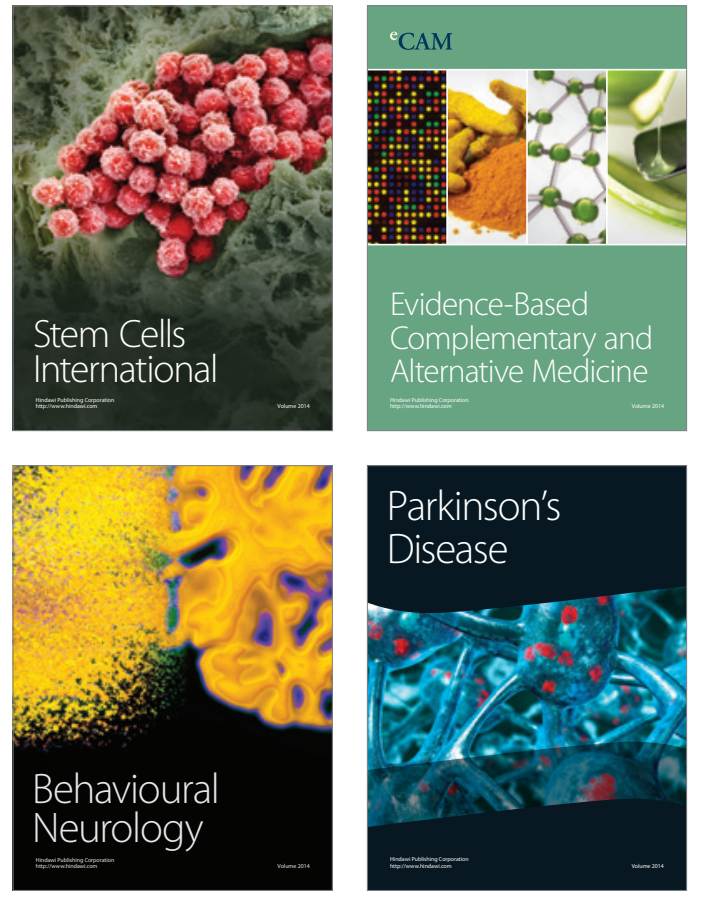
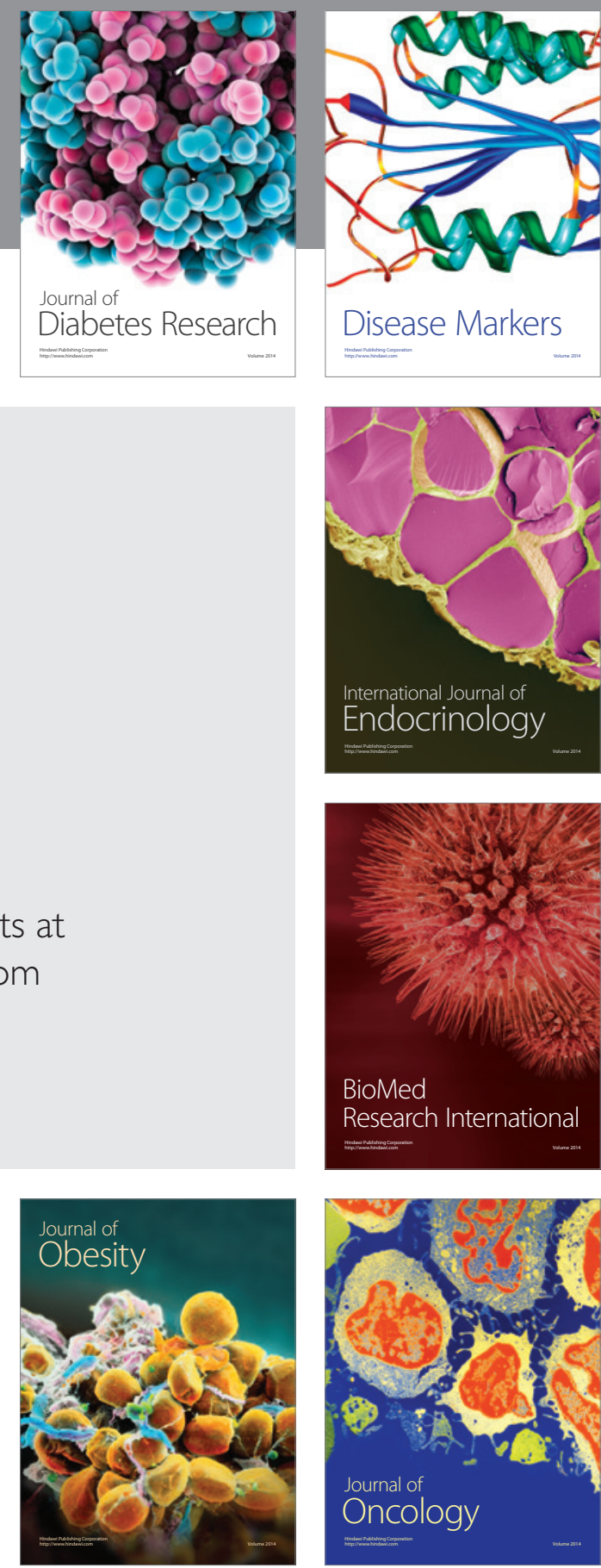

Disease Markers
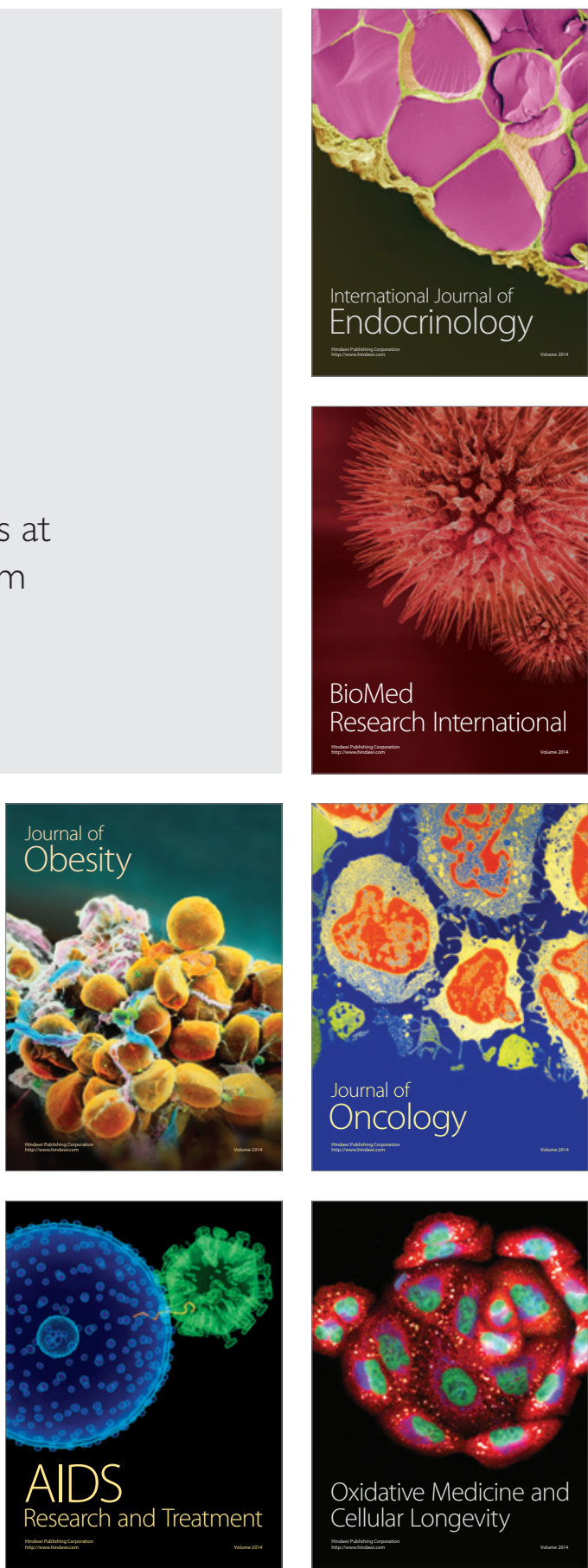